Sādhanā Vol. 40, Part 3, May 2015, pp. 911-923. (C) Indian Academy of Sciences

\title{
Experimental studies on the flow through soft tubes and channels
}

\author{
V KUMARAN \\ Department of Chemical Engineering, Indian Institute of Science, \\ Bangalore 560 012, India \\ e-mail: kumaran@chemeng.iisc.ernet.in
}

MS received 7 July 2014; revised 1 November 2014; accepted 21 November 2014

\begin{abstract}
Experiments conducted in channels/tubes with height/diameter less than $1 \mathrm{~mm}$ with soft walls made of polymer gels show that the transition Reynolds number could be significantly lower than the corresponding value of 1200 for a rigid channel or 2100 for a rigid tube. Experiments conducted with very viscous fluids show that there could be an instability even at zero Reynolds number provided the surface is sufficiently soft. Linear stability studies show that the transition Reynolds number is linearly proportional to the wall shear modulus in the low Reynolds number limit, and it increases as the $1 / 2$ and $3 / 4$ power of the shear modulus for the 'inviscid' and 'wall mode' instabilities at high Reynolds number. While the inviscid instability is similar to that in the flow in a rigid channel, the mechanisms of the viscous and wall mode instabilities are qualitatively different. These involve the transfer of energy from the mean flow to the fluctuations due to the shear work done at the interface. The experimental results for the viscous instability mechanism are in quantitative agreement with theoretical predictions. At high Reynolds number, the instability mechanism has characteristics similar to the wall mode instability. The experimental transition Reynolds number is smaller, by a factor of about 10, than the theoretical prediction for the parabolic flow through rigid tubes and channels. However, if the modification in the tube shape due to the pressure gradient, and the consequent modification in the velocity profile and pressure gradient, are incorporated, there is quantitative agreement between theoretical predictions and experimental results. The transition has important practical consequences, since there is a significant enhancement of mixing after transition.
\end{abstract}

Keywords. Laminar-turbulent transition; soft tubes/channels; hydrodynamic stability; transition; turbulence; internal flows.

\section{Introduction}

Studies on the flow past flexible surfaces can be broadly classified into two types. Studies on external flows past immersed objects have been motivated by the possibility of drag reduction by 
either an increase in the transition Reynolds number, or due to turbulence attenuation. In the case of internal flows, one important motivation has been the enhancement of mixing due to a reduction in the transition Reynolds number. Though the theoretical linear stability studies for these two types of flows are very similar, the treatment of the surface is very different. The experimental set-up used is also very different - whereas studies on external flow usually employ large immersed objects which are dragged through the fluid, the studies in internal flows are often carried out in channels and tubes of sub-millimeter diameter.

The early studies on the flow past flexible surfaces were motivated by marine and aerospace applications, specifically the possibility of reduction in the drag force on air-borne and waterborne vehicles by making the 'skin' of the vehicles soft. One of the inspirations for much of the research in the area is 'Gray's paradox' relating to the efficiency of swimming dolphins (Gray 1936; Fish 2006). Though it has been known since antiquity that dolphins have the ability to swim at high speeds above $10 \mathrm{~m} / \mathrm{s}$, an attempt to examine the drag force of a swimming dolphin was first carried out by Gray (1936) using a rigid model to calculate the power required for swimming. The results of the calculation showed that the power required for the swimming dolphin, assuming that the boundary layer flow around the dolphin is turbulent, was much larger than the muscle power that can be generated. This led to speculation that the dolphin has an ingenious mechanism to maintain a laminar flow, possibly due to special features of the dolphin skin. Though later work (Fish 2006) has cast doubts about the fundamental premise that the power required for swimming at high speeds cannot be generated by dolphin muscles, experimental studies were designed to examine whether the compliance of dolphin skins could cause either transition delay, that is, an increase in the Reynolds number for laminar-turbulent transition or a reduction in the turbulence intensities.

The first experimental studies on internal flows through conduits bounded by soft walls was carried out by Lahav et al (1973) and Krindel \& Silberberg (1979). They conducted experiments in gel-walled tubes of sub-millimeter diameter and found that the flow rate through the tube is smaller than the flow rate expected for a rigid tube for the same pressure difference. This was interpreted as a decrease in the transition Reynolds number in the flow through a soft tube; the latter being considerably lower than that in the value of 2100 for the flow in a rigid tubes. Subsequently, there have been doubts expressed (Yang et al 2000) whether the decrease in the flow rate is due to an instability of the laminar flow, or whether it could be explained by the convergence of the tube due to the applied pressure gradient. However, there is now a large amount of theoretical and experimental work to establish that the original premise of Lahav et al (1973) and Krindel \& Silberberg (1979) is correct, and that the transition in soft tubes and channels could occur at a Reynolds number much lower than that in rigid tubes and channels.

Context for the interpretation of the experimental results is provided by the theoretical studies, based mainly on linear stability calculations of the flow response to infinitesimal perturbations. Though the linear stability procedures for external and internal flows are broadly similar, the treatment of the soft boundary is very different. The theoretical studies on external flows usually consider a boundary layer type base flow. The surface is usually modeled as a spring-backed plate. Surface displacement is permitted normal to the surface, and the surface response is usually modeled by an admittance function which is the ratio of the normal stress and the normal displacement of the surface. Visco-elastic continuum models for boundary layer flows have been used by Yeo (1988); Yeo et al $(1994,2001)$ where the displacement field within the solid wall is modeled using elasticity equations. A linearised form of the elasticity equations is used, where the velocity and stresses in the viscoelastic continuum are modeled as linear functions of the displacement field. These studies recover the Tollmien-Schlichting modes and the flow induced 
surface instabilities observed with the spring-backed plate models, though multi-layering provides additional control over the stability characteristics due to the possibility of adjusting the elasticity moduli of the multiple layers.

Studies on internal flows usually consider the surface as a visco-elastic continuum. The momentum conservation equations within the medium are solved to determine the displacement field using a continuum model, and the displacement field within the medium is coupled to the fluid velocity field using the interface velocity and stress continuity conditions. In contrast to the surface conditions typically employed in external flows, tangential motion at the surface is permitted in internal flows. This leads to modes of destabilisation that are qualitatively different from those in external flows. This distinction is brought out in the present analysis by first providing a brief account of transition in external flows, followed by a comprehensive review of the transition in internal flows.

The transition in internal flows is divided into two regimes, the low Reynolds number regime and the high Reynolds number regime. It has been predicted, and experimentally verified, that there could be a transition even at zero Reynolds number in the flow through a soft channel or tube, provided the shear modulus of the wall of the tube is sufficiently soft. At high Reynolds number, there are two broad instability mechanisms that have been predicted, the inviscid instability in which the flow structure is similar to that for the instability in a rigid channel, and the wall mode instability which is qualitatively different. The mechanism of the low Reynolds number instability and the high Reynolds number wall mode instability, which is the transfer of energy from the mean flow to the fluctuations due to the shear work done at the interface, is qualitatively different from that in a rigid conduit. Experiment confirms the presence of the high Reynolds number wall mode instability in channels and tubes whose characteristic length is less than a millimeter. There is a significant enhancement of mixing after transition, and the mixing time could be orders of magnitude smaller than the molecular diffusion in a laminar flow.

Experimental studies on external flows, and their theoretical context, are briefly reviewed in the next section. In section 3, the low Reynolds number studies of the flow of a viscous fluid past a polymer gel at low Reynolds number is first discussed, followed by high Reynolds number studies in gel-walled channels and tubes. In each section, we highlight the important outstanding issues which provide scope for further work.

\section{External flows}

Kramer (1960a, b, 1962) carried out the first experiments to test whether dolphin skin does reduce drag, based on the hypothesis that the damping due to the viscoelastic nature of the dolphin skin could result in additional dissipation of the turbulent fluctuating energy, and render the flow around the dolphin laminar. In the experiments, an axisymmetric cylindrical object with a rounded end of length about $1 \mathrm{~m}$ was sheathed in an artificial covering with visco-elastic properties similar to a dolphin skin. The object was towed in the ocean at speeds of about $10 \mathrm{~m} / \mathrm{s}$, and the drag force was measured. As a control, the drag was also measured on another object of the same shape without the artificial covering. It was found that the drag on the object with the artificial covering was up to $60 \%$ less than the object without the covering, and the reduction in drag depended on the visco-elastic properties of the covering. As the damping in the flexible surface was increased, the drag force was found to first decrease and then increase, indicating that an optimum damping is necessary for maximum drag reduction. Subsequent experimental studies (Carpenter \& Garrad 1985) were not able to reproduce the extent of damping observed in the original experiments of Kramer, but that could be because the object shapes and the visco-elastic 
parameters used there were different. This seems to suggest that the drag reduction mechanism is very sensitive to the properties of the compliant surface.

Two broad mechanisms of drag reduction have been considered, transition delay (increase in the transition Reynolds number due to the compliant surface) and turbulence reduction. The effect of compliance on the stability of the flow past a flexible surface has been analysed extensively, starting with the pioneering work of Benjamin (1960, 1963) and Landahl (1962). Three modes of instability were identified, the class A modes which are modifications of the TollmienSchlichting modes in the flow past a rigid surface and which are destabilised by increased dissipation in the elastic medium; the class B waves which are modifications of the resonant surface waves on the medium where the wave speed is close to that for the free surface waves on the medium and which are stabilised by increased dissipation, and the class $\mathrm{C}$ or Kelvin-Helmholtz type of modes similar to the aerodynamic flutter which can exist in inviscid flows. Later, Carpenter \& Garrad $(1985,1986)$ simplified the classification into the Tollmien-Schlichting modes (class A of Benjamin and Landahl) and the Flow Induced Surface Instability (class B and $\mathrm{C}$ of Benjamin and Landahl). It was also recognised that the modal coalescence of the TollmienSchlichting modes and the traveling wave flutter could result in a powerful new instability when the free surface wave speed is close to the wave speed of the Tollmien-Schlichting modes. The opposing effects of damping on the class A and class B modes could explain the initial decrease and later increase in the drag force observed by Kramer. On the basis of a detailed analysis of the viscoelastic properties of Kramer-type coatings, Carpenter \& Garrad $(1985,1986)$ concluded that a substantial increase in the transition Reynolds number could be achieved, but that the increase is sensitive to the viscoelastic properties of the surface.

Waves in both laminar and turbulent flows have been observed by Hansen \& Hunston (1974, 1983) in their experiments where a disk coated with a compliant material is rotated in a fluid. They have reported a hydroelastic instability with a very small wave speed, and a standard analysis of a static divergence type instability described the system well in the laminar regime. In the study of Gad-el Hak et al (1985), a flat sheet covered with a layer of soft material was towed through a tank in a turbulent flow. They also reported an instability of the divergence type, and the wave speed depended on the elasticity of the material.

Though the above studies provided a lot of insight into the effect of compliant surfaces on external flows around immersed objects, they do not seem to have been followed up in detail more recently. In particular, it would be of great value to use modern techniques such as Particle Image Velocimetry and laser based surface profile analysis to understand the interaction between the dynamics of the flow and the surface. The models used for the compliant surface have almost uniformly been of the spring-dashpot type models, with a submerged damping fluid. This is appropriate for the dolphin skin models of the Kramer type, but a continuum approximation is more suitable for viscoelastic continua that are used in the experiments of Hansen \& Hunston (1974, 1983) or Gad-el Hak et al (1985). Continuum models are also appropriate for internal flows through conduits bounded by flexible walls, which is the main subject of the present review.

\section{Internal flows}

\subsection{Low Reynolds number}

It is relatively difficult to attain the limit of low Reynolds number in experiments, since it requires very small dimensions and very viscous fluids. For fluids such as water, the Reynolds number is 1 when the characteristic length is $1 \mathrm{~mm}$ and the velocity is of the order of $1 \mathrm{~mm} / \mathrm{s}$, and a smaller length and/or velocity is required to make the Reynolds number much less than 1 . In addition, 
a dynamical fluid-wall interaction is expected only when the dimensionless number $(V \mu / G R)$ is $O(1)$, where $\mu$ is the fluid viscosity, $V$ is the velocity, $G$ is the shear modulus of the wall and $R$ is the characteristic length. For fluids such as water with velocity $1 \mathrm{~mm} / \mathrm{s}$ and characteristic length $1 \mathrm{~mm}$, this dimensionless number is $O(1)$ only when the shear modulus of the elastic material is $10^{-3} \mathrm{~Pa}$. This is impossible to attain with real materials; the elasticity of soft materials such as polyacrylamide gels is, at minimum, about $10^{3} \mathrm{~Pa}$. Lower Reynolds numbers can be attained with higher viscosity fluids. However, when higher viscosity fluids are used to reduce the Reynolds number, the pressure drop required to drive the flow through the conduit increases significantly, linearly proportional to the viscosity and velocity and inversely proportional to the square of the characteristic length. The wall may not have the necessary strength to withstand the large pressures generated, especially if it is made of a soft material. There do not seem to be experiments which have examined the viscous flow $(\mathrm{Re} \ll 1)$ in conduits whose walls are made of a soft material. However, there have been a series of experiments carried out by Kumaran \& Muralikrishnan (2000); Muralikrishnan \& Kumaran (2002) and Eggert \& Kumar (2004) in a commercial rheometer which is used for measuring fluid viscosity. Though this is an open flow, where the fluid is sheared between two parallel plates, the experiments were motivated by theoretical predictions of an instability in channel and tube flows at low Reynolds number (Kumaran et al 1994; Kumaran 1995).

The flat plate geometry of the rheometer consists of a bottom stationary plate and a top plate that rotates, usually with a controlled torque. The fluid sample is placed on the bottom plate, the top plate is lowered to attain a pre-defined thickness, and then the top plate is rotated. The torque and the angular velocity on the top plate are measured, and the stress and strain rate at the outer edge are calculated assuming the flow is laminar.

In the experiments of Kumaran \& Muralikrishnan (2000); Muralikrishnan \& Kumaran (2002) and Eggert \& Kumar (2004), the bottom plate was replaced by a soft poly-acrylamide gel. The gel is very nearly incompressible, and the shear modulus of the gel can be made as low as $1000 \mathrm{~Pa}$ by adjusting the concentration of the cross-linker during the gelation process. The fluid used was silicone oil, which has a viscosity about 1000 times that of water. Consequently, the Reynolds number based on the linear velocity of the top plate at the outer edge was less than $10^{-2}$ in the experiments. The thicknesses of the gels were in the range of 4-6 mm, while the fluid thickness was at most $1 \mathrm{~mm}$. In a typical experiment for measuring viscosity, the rheometer was operated in the stress-controlled mode, the stress is gradually increased and the strain rate recorded. The viscosity is determined as the ratio of the stress and the strain rate. It is important to note that the stress and strain rates are calculated, by the rheometer software, from the torque and angular velocity assuming the flow is laminar. For a Newtonian fluid, such a measurement just provides a viscosity which is independent of the strain rate.

In the experiments of Kumaran \& Muralikrishnan (2000); Muralikrishnan \& Kumaran (2002), it was found that as the stress is increased, the viscosity was a constant until a critical stress where the viscosity showed a sudden and dramatic increase. Since the rheometer was operated in the stress-controlled mode, the increase in viscosity was accompanied by a decrease in the strain rate. The increase was found to be monotonic, and the system did not relax to another steady state, and if continued little bits of gel were found to break off the surface. However, if the experiments were stopped before the gel was altered irreversibly and the experiments were run again, the strain rate for the increase in viscosity was found to be repeatable. The authors interpreted this as the transition of the flow from a laminar flow to a more complicated flow profile at a critical scaled strain rate. The increase in the apparent viscosity is because the rheometer software considers the flow to be laminar while calculating the viscosity. 
The experiments were compared with the theoretical predictions (Kumaran et al 1994). In the low Reynolds number limit, the relevant parameters are the velocity of the top plate $V$, the fluid and gel viscosities $\mu$ and $\mu_{g}$, the shear modulus of the elastic material $G$, the characteristic length scale $R$ which is considered to be the fluid thickness, and the ratio of the gel and fluid thickness $H$. Based on dimensional analysis, it is possible to define a scaled velocity $(V \mu / G R)$, which is a function of the other dimensionless parameters $\left(\mu_{g} / \mu\right)$ and $H$. In the experiments, $G$ was varied between 1000 and $4000 \mathrm{~Pa}$, and the gel viscosity $\mu$ was also determined experimentally. The experimental values for $(V \mu / G R)$ at transition were found to be in quantitative agreement with the theoretical predictions (Kumaran et al 1994). The theoretical prediction of a sub-critical instability (Shankar \& Kumaran 2001b), was also confirmed by the absence of a post-transition nearby steady state in the experiments.

More detailed experiments on the dynamical behaviour of the system were carried out by Eggert \& Kumar (2004) in a similar geometry. They observed that if the stress is raised to a value slightly above the transition value and maintained at that value, the viscosity shows sustained oscillations. Moreover, if the stress is first increased to a value higher than the critical stress and then decreased again below the critical value, then the strain rate does not decrease to the original value, the viscosity does not decrease to the original value but it exhibits sustained oscillations. These results confirm that the transition is sub-critical, but there does not appear to be any theory currently for the post-transition behaviour.

The enhancement of mass transport rates due to the instability was studied by Shrivastava et al (2008). These experiments were performed in a channel with one wall made of a soft polyacrylamide gel. An electrochemical technique was used to measure the mass transfer coefficient, where an electrolyte was pumped into the channel, and the currents were measured using platinum electrodes placed on the cell walls. The dimensionless parameter $(V \mu / G R)$ was maintained $O(1)$ in the experiments, and the enhancement in the mass transfer coefficients, as evidenced from the enhancement in the limiting current, was measured. The authors reported that the mass transfer rates were enhanced up to $25 \%$ in comparison to those for the flow through a rigid channel, and this was attributed to a transition to a more complicated profile due to the low Reynolds number transition induced by a soft wall.

Recent experiments by Neelamegam et al (2013) have been carried out on the rheometer geometry (Kumaran \& Muralikrishnan 2000; Eggert \& Kumar 2004), but have used a viscoelastic fluid instead of a Newtonian fluid in contact with a polymer gel. Here, the objective was to examine the effect of the soft wall on the elastic instability in a rotating flow between two plane surfaces. The authors report that the purely elastic instability in a torsional flow is suppressed due to a soft deformable surface. In the low Reynolds number limit, the critical Weissenberg number (product of the strain rate and the polymer relaxation time) increases as the shear modulus of the gel decreases, or the non-dimensional elasticity parameter $(\mu / G \lambda)$ increases. Here, $\lambda$ is the relaxation time of the viscoelastic fluid, and $\mu$ and $G$ are the fluid viscosity and wall shear modulus respectively. The mechanism appears to be the compression of the gel due to the first normal stress difference, and the consequent decrease in the strain rate due to an increase in the fluid layer thickness. Though experiments on the effect of visco-elasticity on planar flows past a soft deformable surface do not seem to have been performed, theoretical calculations predict that visco-elasticity tends to stabilise the instability due to the fluid-wall coupling (Shankar \& Kumar 2004; Chokshi \& Kumaran 2007).

Even though the experiments have validated the theoretical predictions in some flow regimes, there is much to be done to develop a comprehensive picture of the low Reynolds number instability. Three important outstanding issues are as follows. 
1. The transition at low Reynolds number is found to depend sensitively on the specific form of the constitutive relation for the viscoelastic solid. While the early studies (Kumaran et al 1994; Kumaran 1995) considered a linear elastic solid, later studies of Shankar \& Kumar (2004) and Gkanis \& Kumar (2005) correctly used a frame-invariant strain tensor. Later studies by Chokshi \& Kumaran (2008) found that when the neo-Hookean model is used for the solid wall, the transition Reynolds number increases in comparison to that for a linear model. The details of the wall model were not important for the experiments conducted in a rheometer (Kumaran \& Muralikrishnan 2000; Muralikrishnan \& Kumaran 2002; Eggert \& Kumar 2004) because the ratio of gel and fluid thickness was relatively large in those cases (greater than about 4), and the wall constitutive relations do not significantly affect the transition Reynolds number when the wall thickness is much larger than the fluid thickness. Further experiments are required for the case where wall and fluid thicknesses are comparable, in order to examine the effect of wall constitutive relations on the stability. One important difference that could be compared directly is the difference in the prediction for the dependence of the instability on the ratio of viscosities. The linear model (Kumaran et al 1994) predicts an instability only when the ratio of the gel and fluid thicknesses $H$ is greater than $\sqrt{\mu_{r}}$, where $\mu_{r}$ is the ratio of the gel and fluid viscosities. The new-Hookean model predicts an instability at all values of $H$. Experiments at small values of $H$ would clarify this issue.

2. Even though the instability has been inferred from viscosity measurements, there is as yet no direct visualisation of the instability. This is due to a combination of factors, such as the small dimensions, and the prediction of very high frequencies of the order of a few $100 \mathrm{~Hz}$ for the most unstable modes. It would be desirable to have a direct visualisation of the instability in a manner similar to those of Hansen and Hunston.

3. A more difficult experiment is the verification of the instability in a tube or a channel. The channel flow has been experimentally realised by Shrivastava et al (2008), but detailed measurements on flow transition were not carried out. Studies on a tube flow appear to be less feasible, due to the large pressure difference required, and the consequent large deformation of the tube. However, if such experiments can be carried out, they would serve to distinguish between the linear model (Kumaran 1995) which predicts a transition in the viscous limit, and the neo-Hookean model (Gaurav \& Shankar 2009) where the transition is stabilised.

\subsection{High Reynolds number}

The pioneering experiments in the transition in internal flows at high Reynolds number were carried out by Lahav et al (1973) and Krindel \& Silberberg (1979). Gel-walled tubes of radius $0.15 \mathrm{~mm}$ and length $4 \mathrm{~cm}$ were fabricated within cylindrical polyacrylamide gels of outer diameter 2.6 or $9 \mathrm{~mm}$, by a template assisted method using a hypodermic needle as a template. The ends of the cylinder were sealed, a pressure difference was applied across and the flow rate was measured. The deformation in the shape of the gel could be measured under a microscope, and the authors had also made a provision for introducing a dye stream at the center of the tube and observing the motion of the dye during flow. The shear moduli of the gels were very small, between 0.3 and $1.2 \mathrm{kPa}$.

The authors plotted the ratio of the flow rate $Q$ and the flow rate expected for a laminar flow, $Q_{0}$, as a function of the Reynolds number. The ratio $\left(Q / Q_{0}\right)$ for a rigid tube was equal to 1 at low Reynolds number, but there was a near-discontinuous decrease at the expected transition Reynolds number of about 2100. However, for a soft gel tube, the authors reported that $\left(Q / Q_{0}\right)$ decreases below 1 at a Reynolds number as low as 700 . The authors interpreted this as a transition to turbulence due to wall oscillations. Observations of the motion of the dye-stream at the 
center of the channel indicated that the dye-stream breaks up at a higher Reynolds number than that at which $\left(Q / Q_{0}\right)$ decreases below 1 . The authors interpreted this as an indication that the flow near the walls becomes turbulent due to wall motion even when the flow in the center is laminar. As the Reynolds number is increased, the authors reported break-up of the dye-stream at the center of the channel as well, indicating that the flow in the entire tube is turbulent. A correlation was derived relating the transition Reynolds number to the dimensionless pressure gradient $\left(R_{G}|\nabla p| / 2 G\right)$, where $R_{G}$ is the radius of the gel tube, $|\nabla p|$ is the magnitude of the pressure gradient and $G$ is the shear modulus of the gel. The authors refer to this as the shear strain in the gel, but a cause for concern in interpreting this as a shear strain is that values as large as 10 are reported for this parameter-since polyacrylamide is a brittle material, it is unlikely to sustain strains greater than about 1 . This is consistent with the rather modest change in radius of the inner tube, up to a maximum of about $20 \%$, that is observed in experiments.

A subsequent numerical study (Yang et al 2000) on the flow in a converging tube seems to cast a doubt about the interpretation of the results of Krindel \& Silberberg (1979). Yang et al (2000) studied the flow in a converging tube, and found that the flow acceleration due to the convergence of the streamlines could result in a decrease in the ratio $\left(Q / Q_{0}\right)$. They also derived a modified expression for the $\left(Q / Q_{0}\right)$ which depends on the pressure gradient as well as the shear modulus of the gel. They considered a velocity profile that is a quartic function of the tube radius, a generalisation of the parabolic profile for a laminar flow, and also included the variation in the tube radius using a model for the wall displacement in a cylindrical shell. Using a modified relation between the flow rate and displacement, the authors were able to reproduce the relation between the flow rate and pressure drop in the experiments of Lahav et al (1973) and Krindel \& Silberberg (1979). They interpreted the parameter $\left(R_{G}|\nabla p| / 2 G\right)$ as the slope of the gel wall. Though the results were quantitatively reproduced, systematic improvements are possible for the model of Yang et al (2000). Firstly, the authors seem to have considered only normal wall displacements, whereas in reality the gel will experience both tangential and normal displacements due to the shear stress and pressure. It would be necessary to solve the continuum equations within the gel wall as well, in order to determine the displacements in the axial and radial directions.

Experiments on gel-walled tubes of diameter $800 \mu \mathrm{m}$ and $1200 \mu \mathrm{m}$ and length about $20 \mathrm{~cm}$ were carried out by Verma \& Kumaran (2012). Polydimethyl siloxane (PDMS) gels were used in the experiments, and the catalyst concentration in the gel was varied between $10 \%$ and $1.75 \%$, in order to vary the gel shear modulus between a maximum of $0.5 \mathrm{MPa}$ and a minimum of about $18 \mathrm{kPa}$. The gels were found to be nearly incompressible, since the compression modulus is at least 10 times the shear modulus. The tubes were fabricated within gel blocks using the templateassisted method, where glass rods were used as the templates. The tube was connected to a pressurised water tank through a needle valve to control the flow rate. The tubes were fabricated with a 'development' section of length about $10 \mathrm{~cm}$, where the wall is made of hard gel, to ensure that inlet disturbances are damped and that the flow is parabolic at the entrance to the 'test' section of length about $9.5 \mathrm{~cm}$ made of soft gel. A pressure transducer was used to measure the pressure at the inlet to the test section. A provision was made for injecting a dye-stream at the inlet of the tube, and the progress of the dye-stream was imaged using a camera and microscope. In addition, laser scattering off the curved wall of the tube was used to detect wall motion.

The friction factor was calculated as a function of Reynolds number for tubes with different wall shear moduli in the test section. There is significant wall deformation in the test section made of soft gel in the experiments, though there is very little deformation in the development section made of hard gel. The tube diameter first increases at the entrance of the test section and then shows a gradual decrease downstream as the pressure decreases. The variation in the tube 
radius was incorporated in the friction factor and Reynolds number by defining an average tube radius integrated over the length of the test section.

When the test section is made of hard gel with a shear modulus of about $0.5 \mathrm{MPa}$, the friction factor increases beyond the value of $(16 / \mathrm{Re})$ for a laminar flow at a Reynolds number of about 2000, which corresponds to the transition Reynolds number for a rigid tube. However, as the shear modulus is decreased, the friction factor curve departs from the laminar value of $(16 / \mathrm{Re})$ at a lower Reynolds number indicating that the transition Reynolds number decreases. The transition Reynolds number decreased to a minimum of about 500 for the softest gels with shear modulus $18 \mathrm{kPa}$ and with diameter $800 \mu \mathrm{m}$. The transition was confirmed by the dye-stream and the wall oscillation measurements. In the dye-stream experiments, it was observed that the dye-stream breaks up in the downstream converging section of the tube where the flow is accelerating. The flow appears stable in the upstream diverging section where the slope of the walls is a maximum. An onset of wall oscillations was also observed coinciding with the dye break-up and the increase in the friction factor.

The experimental results were surprising, since it was assumed, for some time after the instabilities were theoretically predicted, that the high Reynolds number instabilities could not be realised in practice. In order to place the high Reynolds number results in context, it is necessary to summarise the salient theoretical studies on the stability of the flow through tubes and channels at high Reynolds number. Here, the transition Reynolds number is expressed as a function of the dimensionless parameter $\Sigma=\left(\rho G R^{2} / \mu^{2}\right)$ which is independent of the flow velocity and depends only on the flow geometry and material properties. Here, $\rho$ and $\mu$ are the fluid density and viscosity, $G$ is the shear modulus of the wall, and $R$ is the characteristic length (tube radius or channel width). In contrast to the low Reynolds number instability, the details of the wall model do not have a significant effect on the instabilities at high Reynolds number (greater than about 10) and it is sufficient to consider a linear wall model. There are two classes of instabilities that have been studied at high Reynolds number, the inviscid modes (Kumaran 1996; Shankar \& Kumaran 1999, 2000) and the wall modes (Kumaran 1998; Shankar \& Kumaran 2001a; Chokshi \& Kumaran 2009).

The inviscid modes are usually a modification of the Tollmien-Schlichting instability in the flow past rigid surfaces, involving an internal critical layer of thickness $\mathrm{Re}^{-1 / 3}$ where the flow velocity is equal to the wave velocity, and it is necessary to include viscous effects even at high Reynolds number. A simple dimensional analysis indicates that the transition flow velocity has to scale as $(G / \rho)^{1 / 2}$ for this mode, and so the transition Reynolds number scales as $\operatorname{Re} \propto \Sigma^{1 / 2}$. Theorems have been derived for the high Reynolds number inviscid flows (Shankar \& Kumaran 2000) in a manner similar to the Rayleigh and Fjortoft theorems for parallel shear flows. These theorems indicate that the parabolic flow in a tube is always stable to axisymmetric disturbances, but could be unstable to non-axisymmetric disturbances. However, the developing flow in a tube and the flow in a converging tube could be unstable to axisymmetric disturbances. In the wall mode instability (Kumaran 1998; Shankar \& Kumaran 2001a; Chokshi \& Kumaran 2009), viscous effects are restricted to a layer of thickness $\mathrm{Re}^{-1 / 3}$ at the wall of the tube. Though these modes are present in rigid tubes as well, they turn out to be always stable. In a flexible tube, the flow does become unstable due to the transfer of energy from the mean flow to the fluctuations due to the shear work done at the surface, and the transition Reynolds number scales as $\Sigma^{3 / 4}$.

The above theoretical predictions are for the instability of flow through a conduit with a soft viscoelastic wall due to a dynamic instability, which is qualitatively different from that in the flow through a rigid channel/tube. In practice, the instability that will be observed at a lower flow velocity is the instability with the lower transition Reynolds number. According to the theoretical predictions, the transition Reynolds number for the flow through conduits with soft walls could be smaller 
than that in rigid conduits only for values of $\Sigma$ which are too low to achieve in practice. For channels/tubes of dimension $100 \mu \mathrm{m}$ to $1 \mathrm{~mm}$, these require a shear modulus of the order of a few tens of Pascals. This is much lower than the minimum shear modulus that can be attained using soft polymer gels, which is a few thousands of Pascals. The parameter $\Sigma$ could be made smaller by either reducing the dimensions or increasing the viscosity of the fluid. However, this would require very large pressure difference of tens of atmospheres across the tube/channel to pump the fluids; it is not feasible to construct microfluidic devices of soft materials which can withstand these large pressures. Due to this, it was considered infeasible to practically realise the high Reynolds number instability at a Reynolds number lower than that for transition in a rigid tube/channel. Due to this, the results of Verma \& Kumaran (2012) for the flow through a soft tube, and Verma \& Kumaran (2013) for a channel flow discussed a little later, were surprising.

In the experiments of Verma \& Kumaran (2012), the transition Reynolds number was found to be lower than theoretical predictions by a factor of 10 . Moreover the scaling of the transition Reynolds number was found to be $\operatorname{Re} \propto \Sigma^{5 / 8}$, in between those for the inviscid and wall modes. The change in the scaling was explained on the basis of the flow modification due to the tube convergence, and the consequent increase in the strain rate at the wall. A more detailed calculation (Verma \& Kumaran 2015) indicated that the quantitative decrease in the Reynolds number is due to the modification in the velocity and pressure profiles caused by the deformation of the wall of the tube. The detailed shape of the tube wall was measured in the experiments. The tube shape was reconstructed in a Computational Fluid Dynamics simulation, and the velocity profiles were determined in the simulations for an imposed pressure difference across the tube. Due to the deformation, the velocity profiles were more plug-like in the downstream converging section with a lower curvature at the center and a higher slope at the wall, while the profiles had a higher curvature at the center and a lower slope at the wall in the diverging section. The pressure gradient was found to deviate significantly from the constant value for a cylindrical tube.

The modified velocity profile and pressure gradient were used in a linear stability analysis of a parallel flow in a tube surrounded by a gel wall. The neo-Hookean model for an incompressible solid was used for the wall of the tube, and the linearised equations were solved for the fluid velocity fluctuations and the displacement fluctuations in the wall. The predictions of the transition Reynolds number in the linear stability analysis are in quantitative agreement with experimental results, and the linear stability analysis correctly predicts that the instability first occurs in the downstream converging section of the tube, and not in the upstream diverging section. Thus, the tube deformation and the flow modification could result in a decrease in the transition Reynolds number by a factor of 10 .

Experiments on transition in a microchannel have been recently carried out (Verma \& Kumaran 2013). The microchannels are made of PDMS gel, in which three walls are made of hard gel with shear modulus $0.5 \mathrm{MPa}$, while the shear modulus of the fourth wall is decreased by decreasing the concentration of cross-linker while preparing the gel. The channels were made of rectangular cross section with heights 100 and $160 \mu \mathrm{m}$ and width $1.5 \mathrm{~mm}$, and the soft wall had a thickness of $2 \mathrm{~mm}$. The channels had a length of about $4 \mathrm{~cm}$, with a developing section of length 0.8 $\mathrm{mm}$ in which all four walls are made of hard gel, and a test section of length $3 \mathrm{~cm}$ in which one of the walls is made of soft gel. A pressure transducer is inserted at the inlet to the soft section to measure the pressure drop across the test section. In order to visualise the flow in the channel, a $\mathrm{Y}$ inlet is used in which coloured fluid is pumped through one inlet and clear fluid through the other inlet. The channel is imaged from above in order to examine the mixing between the two inlet streams, and it is also imaged from the side to determine the channel deformation due to the applied pressure drop. In addition, fluorescent beads are embedded in the wall of the channel, and laser scattering off these beads is used to detect wall motion. 
As in the case of the tube flow, a transition is observed in the channel at a Reynolds number much lower than the transition Reynolds number of 1200 for the flow in a rigid channel, and the transition Reynolds number decreases as the shear modulus of the soft wall decreases. At transition, rapid lateral mixing between the two inlet streams is observed, accompanied by the onset of wall oscillations. The mixing is observed in the downstream converging section of the channel, and not in the upstream diverging section. There is substantial channel deformation, and so it is not possible to define a friction factor for this case. However, the pressure difference across the channel could be compared with the pressure difference predicted for a laminar flow in a channel of the same shape. For this, the detailed shape of the channel was determined from the experiments and reconstructed in a flow simulation. The pressure drop in the simulation, at the same flow rate as those in the experiments, was calculated. It was observed that before transition, there is very good agreement between the experimental results and theoretical predictions. However, after transition, the pressure drop predicted theoretically was lower than that observed in the experiments. This is because the flow in the simulations is assumed to be laminar, whereas there is a transition to a turbulent flow due to a dynamical instability in the experiments.

The transition Reynolds number in the channel flow was also found to be lower, by about an order of magnitude, in comparison to that for the parabolic flow in a two-dimensional channel at the same value of $\Sigma$. The reason was found to be the same for the flow in a tube - the modification of the velocity profile and the pressure gradient due to the deformation of the microchannel. In order to reconcile this difference, the modification in the flow profile and the pressure gradient due to deformation was taken into account in a linear stability analysis of the flow of a Newtonian fluid in a channel with one soft wall. The wall was modeled as a neo-Hookean solid, and the parallel approximation was used for the fluid. The linear stability analysis showed that the flow first becomes unstable in the downstream converging section of the channel, and the transition Reynolds number is in quantitative agreement with experimental results. The qualitative features of the dependence of the transition Reynolds number on the parameter $\Sigma$ are similar to that for a tube flow. An analysis of the eigenfunctions of the most unstable modes revealed that the fluid velocity eigenfunctions are large only near the wall of the channel, indicating that the instability is a wall mode, significantly modified by channel deformation.

Experiments on the efficiency of lateral mixing were also carried out by separating the outlets into two streams, and examining a the concentration of a solute which is pumped into only one of the inlet streams. The instability leads to rapid mixing between the two streams, and the mixing time is lower, by five orders of magnitude, in comparison to the diffusive mixing in a laminar flow. Thus, this phenomenon could be a useful means for enhancing mixing in microfluidic devices.

Considerable progress has been made, both theoretically and experimentally, in understanding the transition in the flows through flexible tubes and channels at high Reynolds number. However, there is considerable scope for further work. Some important avenues for future work are as follows.

1. All of work done so far involves considerable deformation of the channel or tube due to the applied pressure drop. To get a clear validation of theories, it is of importance to demonstrate transition in channels or tubes when there is not much wall deformation. This can possibly be achieved by increasing the dimension and decreasing the shear modulus, so that the pressure drop decreases for a fixed Reynolds number.

2. All instabilities observed so far seem to be of the wall mode type. Inviscid modes do not seem to have been observed in experiments. It would be useful to probe parameter regimes where 
inviscid instabilities have a lower transition Reynolds number in comparison to wall mode instabilities.

3. The nature of the flow after transition is an important question. Specifically, whether the structure of the flow (wall layer, buffer layer and core) is different in case there is a dynamical coupling between the fluid and wall dynamics. This could have an effect not just on the flow dynamics, but also on transport processes through the walls of channels and tubes in physiological flows.

\section{Acknowledgement}

The author would like to thank the Department of Science and Technology, Government of India, for financial support. Instructive discussions with Prof. V. Shankar are gratefully acknowledged.

\section{References}

Benjamin T 1960 Effects of a flexible boundary on hydrodynamic stability. J. Fluid Mech. 9: 513-532

Benjamin T 1963 The three-fold classification of unstable disturbances in flexible surfaces bounding an inviscid flow. J. Fluid Mech. 16: 436-450

Carpenter P and Garrad A 1985 The hydrodynamic stability of the flow over kramer-type compliant surfaces. part 1: Tollmien-schlichting instability. J. Fluid Mech. 155: 465-510

Carpenter P and Garrad A 1986 The hydrodynamic stability of the flow over kramer-type compliant surfaces. part 2: Flow induced surface instability. J. Fluid Mech. 170: 199-232

Chokshi P and Kumaran V 2007 Stability of the viscous flow of a polymeric fluid past a flexible surface. Phys. Fluids 19: 034,102

Chokshi P and Kumaran V 2008 Weakly nonlinear analysis of viscous instability in flow past a neo-hookean surface. Phys. Rev. E 77: 056,303

Chokshi P and Kumaran V 2009 Weakly nonlinear stability analysis of a flow past a neo-hookean solid at arbitrary reynolds numbers. Phys. Fluids 21: 014,109

Eggert M and Kumar S 2004 Observations of instability, hysteresis and osciallations in low-reynoldsnumber flow past polymer gels. J. Colloid Int. Sci. 274: 238-242

Fish F 2006 The myth and reality of gray's paradox: Implication of dolphin drag reduction for technology. Bioinsp. Biomim. 1: R17-R25

Gad-el Hak M, Blackwelder R and Riley J 1985 On the interaction of compliant coatings with boundary layer flows. J. Fluid Mech. 140: 257-280

Gaurav and Shankar V 2009 Stability of fluid flow through deformable neo-hookean tubes. J. Fluid Mech. 627: $291-322$

Gkanis V and Kumar S 2005 Stability of pressure driven creeping flows in channels lined with a nonlinear elastic solid. J. Fluid Mech. 524: 357-375

Gray J 1936 Studies in animal locomotion: Vi. The propulsive powers of the dolphin. J. Exp. Biol. 13: 192199

Hansen R and Hunston D 1974 An experimental study of fluid turbulence over compliant surfaces. J. Sound Vib. 34: 297-308

Hansen R and Hunston D 1983 Fluid-property effects on flow-generated waves on compliant surfaces. J. Fluid Mech. 133: 161-177

Kramer M 1960a Boundary layer stabilization by distributed damping. J. Am. Soc. Nav. Eng. 72: 25-33

Kramer M 1960b The dolphins' secret. New Sci. 7: 1118-1120

Kramer M 1962 Boundary layer stabilisation by distributed damping. J. Am. Soc. Nav. Eng. 74: 341-348

Krindel P and Silberberg A 1979 Flow through gel-walled tubes. J. Colloid Int. Sci. 71: 39-50

Kumaran V 1995 Stability of the viscous flow of a fluid through a flexible tube. J. Fluid Mech. 294: 259281 
Kumaran V 1996 Stability of an inviscid flow in a flexible tube. J. Fluid Mech. 320: 1-17

Kumaran V 1998 Stability of wall modes in a flexible tube. J. Fluid Mech. 362: 1-15

Kumaran V and Muralikrishnan R 2000 Spontaneous growth of fluctuations in the viscous flow of a fluid past a soft interface. Phys. Rev. Lett. 84: 3310-3313

Kumaran V, Fredrickson G and Pincus P 1994 Flow induced instability at the interface between a fluid and a gel at low reynolds number. J. Phys. France II 4: 893-911

Lahav J, Eliezer N and Silberberg A 1973 Gel-walled cylindrical channels as models for the microcirculation: Dynamics of flow. Biorheology 10: 595-604

Landahl M 1962 On the stability of a laminar incompressible boundary layer over a flexible surface. $J$. Fluid Mech. 13: 609-632

Muralikrishnan R and Kumaran V 2002 Experimental study of the instability of the viscous flow past a flexible surface. Phys. Fluids 14: 775-780

Neelamegam R, Shankar V and Das D 2013 Suppression of purely elastic instabilities in the torsional flow of a viscoelastic liquid past a soft solid. Phys. Fluids 25: 124,102

Shankar V and Kumar S 2004 Instability of viscoelastic plane couette flow past a deformable wall. $J$. Non-Newtonian Fluid Mech. 116: 371

Shankar V and Kumaran V 1999 Stability of non-parabolic flows in a flexible tube. J. Fluid Mech. 395: 211-236

Shankar V and Kumaran V 2000 Stability of non-axisymmetric modes in a flexible tube. J. Fluid Mech. 407: 291-314

Shankar V and Kumaran V 2001a Asymptotic analysis of wall modes in a flexible tube revisited. Eur. Phys. J. B 19: 607-622

Shankar V and Kumaran V 2001b Weakly nonlinear stability of viscous flow past a flexible surface. $J$. Fluid. Mech. 434: 337-354

Shrivastava A, Cussler E and Kumar S 2008 Mass transfer enhancement due to a soft boundary. Chem. Eng. Sci. 63: 4302-4305

Verma M and Kumaran V 2015 Stability of the flow in a soft tube deformed due to an applied pressure gradient. Phys. Rev. E 91: 043001

Verma M and Kumaran V 2012 A dynamical instability due to fluid-wall coupling lowers the transition reynolds number in the flow through a flexible tube. J. Fluid Mech. 705: 322-347

Verma M and Kumaran V 2013 A multifold reduction in the transition reynolds number, and ultra-fast mixing, in a micro-channel due to a dynamical instability induced by a soft wall. J. Fluid Mech. 727: 407-455

Yang C, Muggeridge Grattoni A CA and Zimmerman R 2000 A model for steady laminar flow through a deformable gel-coated channel. J. Collid Int. Sci. 226: 105-111

Yeo K 1988 The stability of boundary layer flows over single- and multi-layer viscoelastic walls. J. Fluid Mech. 196: 359-409

Yeo K, Khoo B and Chong W 1994 The linear stability of boundary-layer flow over compliant walls: The effect of boundary layer growth. J. Fluid Mech. 280: 199-225

Yeo K, Khoo B and Chong W 2001 Turbulent boundary layer over a compliant wall: Absolute and convective instabilities. J. Fluid Mech. 449: 141-168 